ARTICLE

\title{
OsAAP6 functions as an important regulator of grain protein content and nutritional quality in rice
}

Bo Peng ${ }^{1, \star}$, Huili Kong ${ }^{1, \star}$, Yibo Li $^{1}$, Lingqiang Wang ${ }^{1}$, Ming Zhong ${ }^{1}$, Liang Sun ${ }^{1}$, Guanjun Gao ${ }^{1}$, Qinglu Zhang ${ }^{1}$,

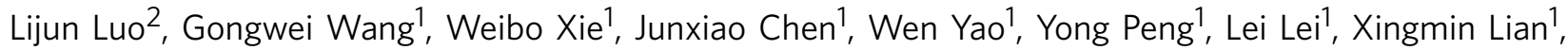
Jinghua Xiao', Caiguo $\mathrm{Xu}^{1}$, Xianghua $\mathrm{Li}^{1} \&$ Yuqing $\mathrm{He}^{1}$

Grains from cereals contribute an important source of protein to human food, and grain protein content (GPC) is an important determinant of nutritional quality in cereals. Here we show that the quantitative trait locus (QTL) $q P C 1$ in rice controls GPC by regulating the synthesis and accumulation of glutelins, prolamins, globulins, albumins and starch. $9 P C 1$ encodes a putative amino acid transporter OSAAP6, which functions as a positive regulator of GPC in rice, such that higher expression of OsAAP6 is correlated with higher GPC. OsAAP6 greatly enhances root absorption of a range of amino acids and has effects on the distribution of various amino acids. Two common variations in the potential cis-regulatory elements of the OsAAP6 5'-untranslated region seem to be associated with GPC diversity mainly in indica cultivars. Our results represent the first step toward unravelling the mechanism of regulation underlying natural variation of GPC in rice.

\footnotetext{
${ }^{1}$ National Key Laboratory of Crop Genetic Improvement and National Centre of Plant Gene Research (Wuhan), Huazhong Agricultural University, Hongshan District, Wuhan 430070, China. ${ }^{2}$ Shanghai Agrobiological Gene Center, Shanghai 201106, China. ${ }^{\star}$ These authors contributed equally to this work.

Correspondence and requests for materials should be addressed to Y.H. (email: yqhe@mail.hzau.edu.cn).
} 
E nhancing nutritional quality of cereals is a means of improving human nutrition and health ${ }^{1}$. Rice, wheat, maize and barley contain different contents of grain protein, which is an important part of total protein in human food ${ }^{2}$. Nearly one-third of the world population currently suffers from protein malnutrition and some diseases are caused by poorquality protein and lack of vitamins and other micronutrients ${ }^{3}$. Over $90 \%$ of rice grain consists of starch and proteins ${ }^{4,5}$ and grain protein content (GPC) is a key factor determining the nutritional quality $^{6}$. Rice is generally recognized as having the lowest GPC of the cereal grains, but net protein utilization from rice is highest among the cereal grains ${ }^{7}$. Moreover, the demand for high-quality cereals as a source of protein is expected to increase dramatically in the near future ${ }^{4}$.

The amino acid composition in rice grain is relatively balanced and the grain proteins are divided into albumins, globulins, prolamins and glutelins ${ }^{8}$. GPC in rice grain is mainly constituted of glutelins and prolamins; glutelins have high nutritive value for the human diet ${ }^{6,9}$. Thus, GPC is an attractive target for enhancing nutritional quality of rice grain. As GPC in rice is determined by complex multigene families and highly polymorphic mixtures of polypeptides, even mutations in a few structural genes have little effect on $\mathrm{GPC}^{8,10,11}$. Interestingly, some genes not only regulate starch storage but also have effects on protein in the endosperm, for example, transgenic RNA interference (RNAi) targeting the Opaque7 gene causes downregulation of GPC in both maize and rice, and the FLOURY ENDOSPERM2 gene mutant results in reduced accumulation of glutelin ${ }^{12,13}$. Although gpal regulates storage protein trafficking, GPC in a rice gpal mutant is the same as in wild-type plants ${ }^{14}$. Many genes/regulators affecting GPC have been isolated from various mutants in cereals ${ }^{12-16}$ and numerous quantitative trait loci (QTLs) for GPC have been detected in genetic mapping populations ${ }^{17-22}$. However, no QTL underlying natural variation and controlling GPC in rice has been cloned, and little is known about how GPC is regulated quantitatively.

Our previous studies revealed a relatively strong QTL cluster controlling $\mathrm{GPC}^{22}$. Several loci within the cluster encode putative amino acid transporters ${ }^{23}$ and the RM472-RM104 region on the long arm of chromosome 1 exhibits the highest value $(32.4 \%)$ of trait variation explained by one QTL among the clusters ${ }^{22}$. Here we report the cloning of the major QTL $q P C 1$ (a putative amino acid permease, OsAAP6) controlling natural variation in GPC by a map-based cloning strategy. We show through overexpression, complementation, RNAi and transmission electron microscopy studies that OsAAP6 functions as an important regulator of GPC and nutritional quality in rice.

\section{Results}

Mapping the $q P C 1$ locus to a $6.7-\mathrm{kb}$ region. To identify the gene(s) underlying the $q P C 1$ QTL for GPC, we used 190 recombinant inbred lines (RILs) derived from a cross between Zhenshan 97 (ZS97, Oryza sativa L. ssp. indica) and Nanyangzhan (NYZ, O. sativa L. ssp. japonica). qPC1 was mapped to the interval between markers RM472 and RM104 on the long arm of chromosome 1 (Fig. 1a). The allele from ZS97 contributed to increased GPC and total amount of essential amino acids ${ }^{22}$.

To fine map the qPC1 locus, we backcrossed RIL-105 (one of the RILs containing the chromosome segment RM472-RM104 from NYZ and $78 \%$ of the genetic background of ZS97) three times $\left(\mathrm{BC}_{3}\right)$ to $\mathrm{ZS} 97 . \mathrm{BC}_{3} \mathrm{~F}_{1}$ heterozygous plants were selfpollinated to develop two $\mathrm{BC}_{3} \mathrm{~F}_{2}$ populations consisting of 6,000 (population 1) and 4,008 (population 2) individuals, and to establish a pair of near-isogenic lines (NILs), NIL(ZS97) and
NIL(NYZ). Analysis of a $\mathrm{BC}_{3} \mathrm{~F}_{2}$ population of 320 individuals derived by self-pollination of a $\mathrm{BC}_{3} \mathrm{~F}_{1}$ heterozygote showed that GPC in heterozygous plants was significantly lower than in ZS97 homozygotes but higher than in NYZ homozygotes (Supplementary Table 1), indicating that the ZS97 high-protein content allele of $q P C 1$ was partially dominant.

Fifty-two recombinants between RM472 and RM104 were identified in population 1 and their genotypes at the $q P C 1$ locus were deduced by progeny testing. Using these data, we mapped $q P C 1$ to the interval between PB2 and PB7 (Fig. 1b). Seven recombinants between $\mathrm{PB} 2$ and $\mathrm{PB} 7$ were detected in population 2 (Fig. 1c). Using markers PB8-PB15, we identified six and two recombinant plants in the intervals PB11-PB12 and PB14-PB15 in the two populations, respectively (Fig. 1c,d). Each recombinant was further phenotyped by progeny testing to deduce the genotype of $q P C 1$ (for example, see Supplementary Table 2). The $q P C 1$ locus co-segregated with marker PB13, located between PB14 and PB15 (Fig. 1c,d).

To convert the genetic map location of $q P C 1$ into a physical map location, we placed the molecular markers onto the physical map of Nipponbare, the reference cultivar for rice genomics. As shown in Supplementary Fig. 1, the PB10-PB15 region was in the same order on the physical map of Nipponbare and on the genetic map derived from the ZS97 $\times$ NYZ population (Fig. 1c). In the Nipponbare genome, the distance between PB14 and PB15 is $6.7 \mathrm{~kb}$ (Supplementary Fig. 1).

Analysis of the genomic DNA data revealed only one predicted gene (NCBI accession no. Loc_Os01g65670) in the $6.7 \mathrm{~kb}$ region and the gene has three predicted full-length complementary DNA (cDNA) sequence (AK121636, AK065286 and AK119529). The sequence of AK121636 includes the full-length cDNA sequence of AK065286 and AK119529, and all of them encode the same amino acid sequence. Thus, this gene (Loc_Os01g65670) was considered the candidate gene for $q P C 1$ (Supplementary Fig. 1). Alignment of the full-length cDNA sequence of AK121636 with the genomic sequence of Nipponbare indicated that $q P C 1$ encodes a putative amino acid transporter belonging to the APC superfamily and has a PF01490 consensus domain (http:// pfam.janelia.org/). Phylogenetic analysis of the qPC1 protein revealed that it is highly homologous to the amino acid permeases (AAPs) family (Supplementary Fig. 2). Interestingly, $q P C 1$ corresponds to OsAAP6 (Loc_Os01g65670), which is highly expressed in seeds and belonging to OsAAT family in rice ${ }^{23}$.

Confirmation of the $q P C 1$ QTL. Three transformation constructs were prepared to confirm the candidate gene (OsAAP6 (ref. 23)) for the $q P C 1$ QTL. First, a construct for overexpression (OX) contained the coding region of OsAAP6 from ZS97 (high GPC) driven by the CaMV $35 \mathrm{~S}$ promoter inside the vector pCAMBIA1301 (Fig. 1e); second, a complementation construct $(\mathrm{ZpZc})$ with the promoter and coding regions of OsAAP6 from ZS97 inserted into pCAMBIA1301S (Fig. 1f); and third, an RNAi construct containing a 580-bp PCR fragment from the fourth exon of OsAAP6 inserted into dspCAMBIA1301 in both the sense and antisense orientations (Fig. 1g). Agrobacterium-mediated transformation was used to introduce OX into NYZ (OX(NYZ)) and Zhonghua 11 (ZH11, OX(ZH11)), ZpZc into NYZ (ZpZc(NYZ)), and RNAi into ZS97 (RNAi(ZS97)) and ZH11 $(\mathrm{RNAi}(\mathrm{ZH} 11))$.

All transgene-positive individuals of OX(NYZ), OX(ZH11) and $\mathrm{ZpZc}(\mathrm{NYZ})$ in the $\mathrm{T}_{0}$ generation had a higher GPC than transgene-negative individuals (Supplementary Fig. 3). Co-segregation analysis between the genotypes and phenotypes in $\mathrm{T}_{1}$ progenies confirmed these effects: all transgene-positive plants of $\mathrm{OX}(\mathrm{NYZ}), \mathrm{OX}(\mathrm{ZH} 11)$ and $\mathrm{ZpZc}(\mathrm{NYZ})$ had higher GPC 

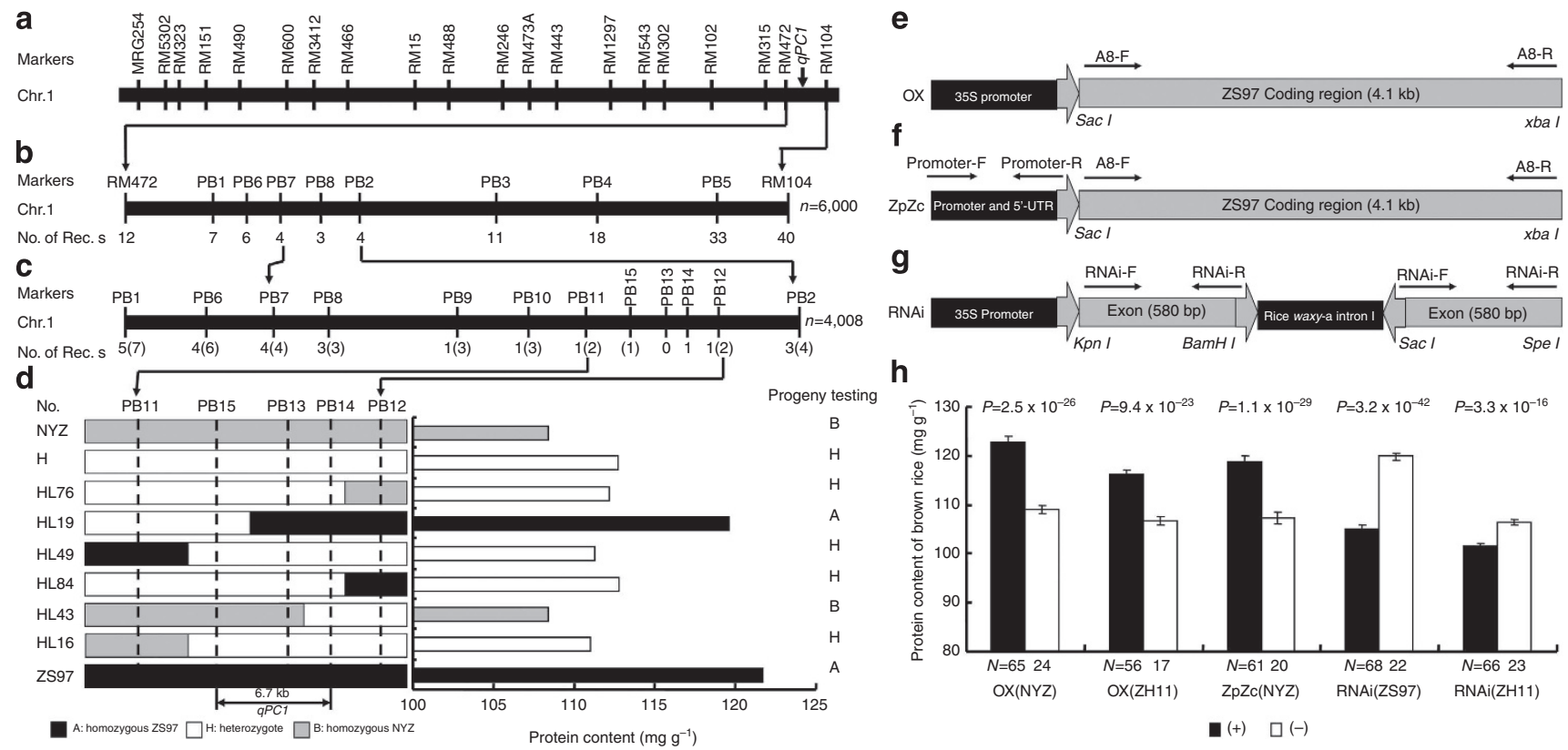

${ }^{n}$

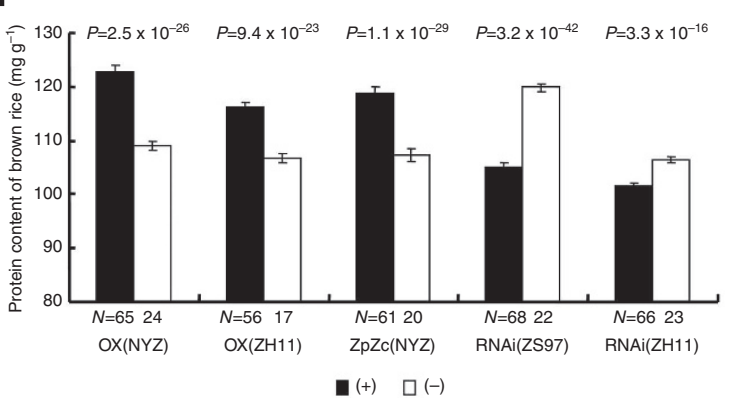

Figure 1 | Map-based cloning of the qPC1 QTL and grain protein content of transgenic plants in $\mathbf{T}_{\mathbf{1}}$. (a) Location of the $q P C 1 \mathrm{QTL}$ on the genetic linkage map of chromosome 1. (b,c) Fine mapping of the qPC1 region using two mapping populations, with 6,000 and 4,008 plants, respectively. No. of Recs, number of recombinants between $q P C 1$ and indicated molecular marker (b). Numbers in parentheses in c correspond to the numbers of recombinants in the b. (d) Genotypes and phenotypes of the recombinants. The phenotype of each recombinant was determined by progeny testing (Supplementary Table 2). (e) The OsAAP6 coding region of ZS97 was inserted into the vector pCAMBIA1301S under control of the CaMV 35S promoter to prepare the overexpression construct (OX). Arrows represent the direction of PCR primers. (f) The 1.8-kb promoter fragment and 5'-UTR of OsAAP6 from ZS97 with its own coding region was inserted into the vector pCAMBIA1301 to prepare the complementation construct (ZpZc). Arrows represent the direction of PCR primers. (g) The 580 bp cDNA fragment from the fourth exon of OsAAP6 was inserted into the dspCAMBIA1301 vector to generate the RNAi construct. Arrows represent the direction of PCR primers. (h) Grain protein contents of transgenic plants in $\mathrm{T}_{1} . \mathrm{N}$, number of plants; $(+)$ and $(-)$ indicate transgene-positive and transgene-negative plants, respectively. P-values were produced by two-tailed t-test. Error bars, s.e.m.

than the untransformed controls (Fig. 1h), whereas lower GPC was observed for RNAi(ZS97) and RNAi(ZH11) in both $\mathrm{T}_{0}$ (Supplementary Fig. 3) and the $\mathrm{T}_{1}$ (Fig. 1h). Co-segregation analysis of genotypes and expression levels with phenotypes in $\mathrm{T}_{2}$ progenies confirmed that GPC was significantly $(P<0.01)$ and positively correlated with expression level of OsAAP6 in all three kinds of transformants, OX(NYZ), ZpZc(NYZ) and RNAi(ZS97) (Supplementary Table 3). Transgenic plants showing higher levels of OsAAP6 expression produced larger amounts of grain storage protein (Supplementary Table 3 and Table 1). Thus, our results confirm that the candidate gene (OsAAP6) is the qPC1 QTL.

Expression pattern of OsAAP6 and subcellular localization. Sixteen tissues from the NILs were assayed for temporal and spatial expression patterns of OsAAP6 by quantitative reverse transcription-PCR (Fig. 2a). OsAAP6 transcripts were detected in all examined tissues and were most abundant in the endosperms at 5-12 days after flowering (DAF) (Fig. 2a), the same timeframe as reported for transcripts of OsAAP6 (ref. 23), and there were significantly higher transcript levels in the endosperms of NIL(ZS97) than in endosperm of NIL(NYZ) (Fig. 2a).

We next examined the expression of the $\beta$-glucuronidase (GUS) gene under control of the OsAAP6 promoter from ZS97 in transgenic plants. All tissues exhibited GUS activity and the GUS signal was particularly strong in vascular tissues of the hull, root, pulvinus, internode, node, seed and endosperm at 10 and $25 \mathrm{DAF}$ (Fig. 2b-m). GUS activity in the endosperm was more abundant in the ovular vascular trace, ovular vascular trace end and lateral stylar vascular traces than in other positions during grain filling (Fig. 2b). Histochemical GUS studies showed that OsAAP6 was expressed mainly in the root rhizodermis and phloem (Fig. 2l,m).
This feature of OsAAP6 closely resembles Arabidopsis AAP family genes and $L H T 1$, which are preferentially expressed in vascular tissues and the root rhizodermiss ${ }^{24-27}$.

To investigate the subcellular localization of OsAAP6 protein, we transiently co-expressed OsAAP6 fused to green fluorescent protein (GFP) with various subcellular markers in rice protoplast $^{28-31}$. Co-expression of OsAAP6-GFP and SCAMP1RFP clearly showed that OsAAP6 was not localized at the plasma membrane or on the trans-Golgi network (Fig. 2n). However, OsAAP6-GFP and the endoplasmic reticulum (ER) marker BiP-RFP overlapped completely when co-expressed in rice protoplasts (Fig. 20). Therefore, OsAAP6 is localized on the $\mathrm{ER}$, but not on the plasma membrane or in the trans-Golgi network.

Genetic variation in the regulatory region of OsAAP6. To identify the sequence variation between the ZS97 and NYZ alleles of OsAAP6, we compared genomic clones from both parents (Fig. 3a). There were eight nucleotide differences between the two varieties in the open reading frame: seven polymorphisms in the first intron and one synonymous mutation (at 3,813 bp) in the fourth exon (Fig. 3a). In addition, 15 polymorphisms between ZS97 and NYZ were revealed in the $5^{\prime}$-untranslated region ( $5^{\prime}$-UTR) and promoter region $(\sim 1.8 \mathrm{~kb})$. These polymorphisms included a 6-bp indel and 14 upstream single-nucleotide polymorphisms (Fig. 3a). Several types of putative regulatory elements were identified within the polymorphic regions of the OsAAP6 promoter and $5^{\prime}$-UTR, which harbours several cis-regulatory elements involved in transcriptional responses, such as a copperresponse element, inr element, ARR1-binding element and other cis-elements (Supplementary Table 4). 
Table 1 | Grain quality traits in NILs and transgenic plants in a 2-year field trial.

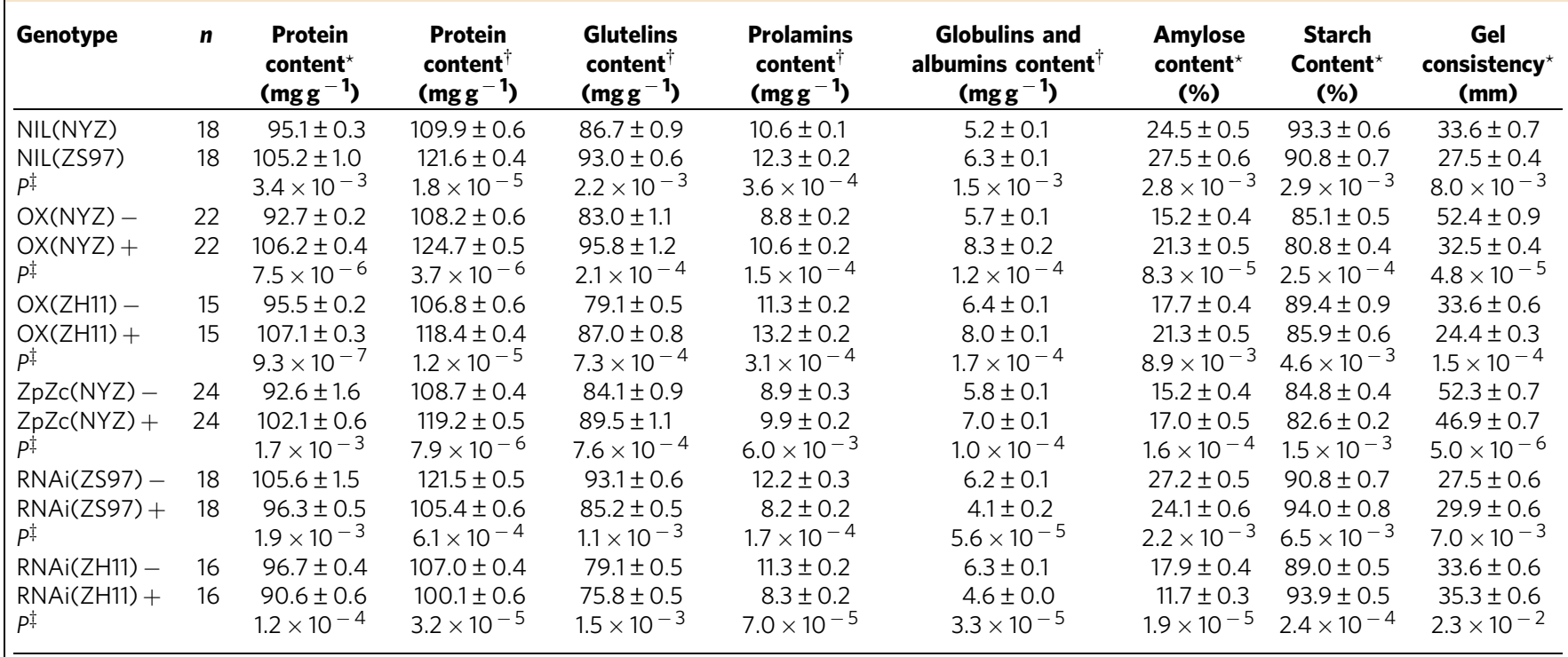

NIL, near-isogenic line; $n$, number of plants with the same genotype.

Data of all the materials are based on field trials using randomized block designs with three replications. All data are means \pm s.e.m. $(+)$ and $(-)$, transgene-positive and negative plants, respectively, and their data are the averages of 2 years. At least 300 grains of each plant were measured for all grain quality traits.

${ }^{*}$ Milled rice was used to determine protein content, starch content, amylose content and gel consistency.

†Brown rice was used to determine the protein content.

$\ddagger P$-values are produced by Duncan's test.

We sequenced the OsAAP6 $5^{\prime}$-UTRs and promoter regions $(\sim 1.8 \mathrm{~kb})$, and the coding regions containing the synonymous mutation site, and measured GPC in 197 accessions of the rice mini-core collection originating from a wide geographic range across Asia (Supplementary Table 5). The collection comprised two subpopulations (Sub1 and Sub2) identified as previous population structure analyses ${ }^{32,33}$. Based on the nucleotide polymorphisms identified between the two parents (Fig. 3a), the sequences of the cultivated varieties were divided into eight haplotypes (Fig. 3b), placed into two groups (A and B) by phylogenetic analysis of the sequences. Five haplotypes were present in Group A (named types 1-5) and three in Group B (types 6-8) (Fig. 3c and Supplementary Table 5). Sub1 with 94 accessions in Group A and 14 accessions in Group B comprised mainly indica cultivars, and Sub2 with 31 accessions in Group A and 58 accessions in Group B were mainly japonica (Fig. 3b,c). Cultivars in Sub1 carrying Group A haplotypes tended to show higher OsAAP6 expression levels in endosperms at $5 \mathrm{DAF}$ and a higher GPC than those having the class B haplotypes (Fig. 3d,e). We also assayed the transcript abundance of OsAAP6 in endosperms at $5 \mathrm{DAF}$ (Fig. 3e) and calculated the correlation between OsAAP6 expression levels and GPC in the indica group. The highly significant correlation $(0.65, P<0.01)$ strongly suggests that expression levels for this gene might be the cause of natural variation in indica GPC. Furthermore, three common nucleotide polymorphisms upstream of the translation start site were identified between Groups A and B (Fig. 3b), and two of the common nucleotide changes $(-7$ to $-12 \mathrm{bp},-32 \mathrm{bp}$ ) in the $5^{\prime}$-UTR were in three potential cis-regulatory elements (copperresponsive element, inr element and sulphur-responsive element) (Supplementary Table 4), which are targets for transcriptional activators and a regulator containing an SBP domain, and are involved in a broad range of responses ${ }^{34-40}$. Consequently, our results imply that the two common variations in the three potential cis-elements of the OsAAP6 5'-UTR seem to be associated with GPC diversity in the Sub1 population (mainly indica cultivars).
A series of $5^{\prime}$-end deletions of the OsAAP6 promoter from ZS97 and NYZ were fused to the GUS gene (Fig. 3f), and their ability to drive the reporter gene was assessed relative to the full-length ZS97 promoter in transgenic plants. Compared with fragments of $-377,-698,-1,226$ and $-1,814$ bp from NYZ, quantitative analysis revealed that the GUS expression levels from ZS97 were significantly increased in young panicles 2 days before flowering and in grains at 5 DAF (Fig. $3 \mathrm{~g}$ ). However, compared with fragments of $-698 \mathrm{bp}$ in grains at $5 \mathrm{DAF}$, the GUS expression levels from the fragments of $-377 \mathrm{bp}$ from ZS97 and NYZ were markedly decreased $(P<0.01)$, respectively (Fig. 3g). Thus, the minimal region $(-698$ to +1$)$ of OsAAP6 may play an important role in regulating the OsAAP6 expression difference between the alleles from ZS97 and NYZ.

Effects of OsAAP6 on grain nutritional quality. To quantify the effects of OsAAP6 on grain quality, we first assayed the quality traits of the NILs and transgenic plants of OX(NYZ), OX(ZH11), ZpZc(NYZ), RNAi(ZS97) and RNAi(ZH11) using data from field trials (Table 1). NIL(ZS97) and transgene-positive plants of $\mathrm{OX}(\mathrm{NYZ}), \mathrm{OX}(\mathrm{ZH} 11)$ and $\mathrm{ZpZc}(\mathrm{NYZ})$ had substantially increased GPC (grain storage proteins, including glutelins, prolamins, globulins and albumins) and amylose contents, coupled with markedly reduced starch content and gel consistency, whereas the reverse was true for RNAi(ZS97) and RNAi(ZH11) (Table 1). There were no significant differences between the NILs and transgenics in other agronomic traits (Supplementary Fig. 4 and Supplementary Table 6).

The total amino acid contents of grains were also determined for the NILs and the transgenic plants. Compared with the corresponding levels in NIL(NYZ) and transgene-negative OX(NYZ) plants, the levels of alanine, leucine, valine, proline, arginine, acidic amino acids and total content of amino acids were significantly increased in NIL(ZS97) and transgene-positive plants, whereas the reverse was true for RNAi (ZH97) 


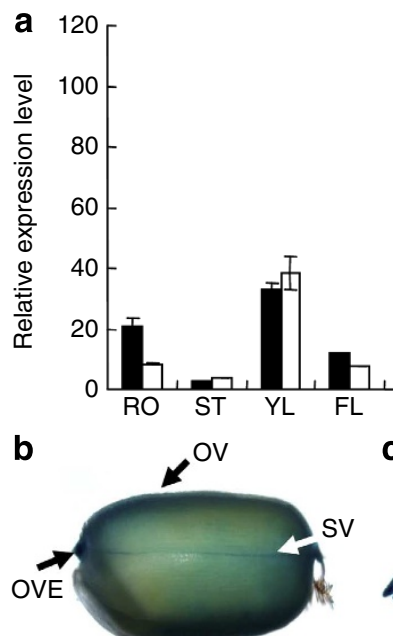

— NIL (ZS97) NIL (NYZ)

f
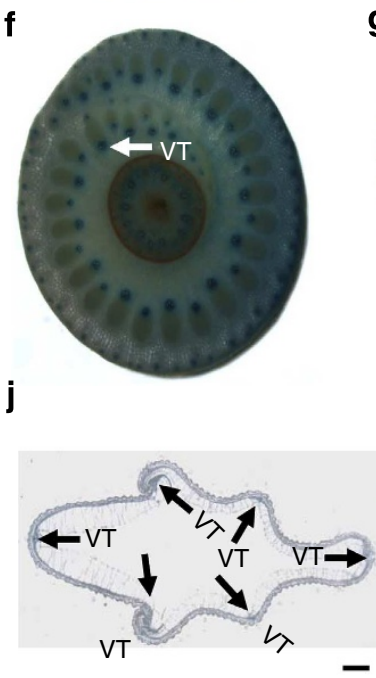

n OSAAP6-GFP
C

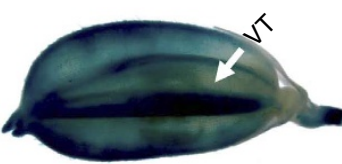

g

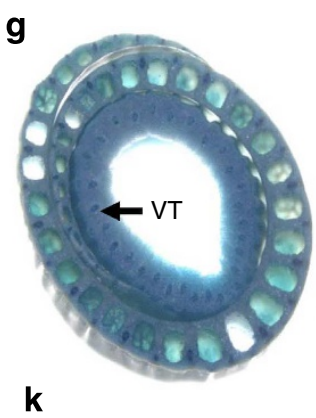

k

I h

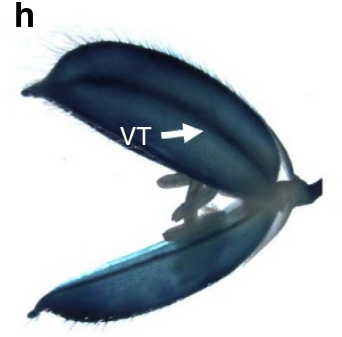

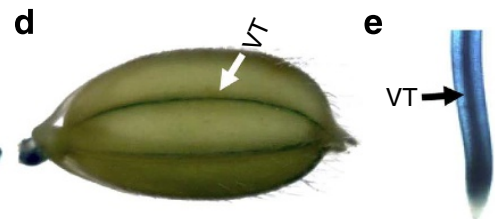

i

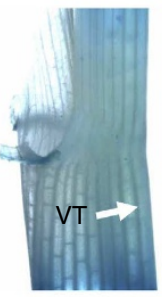

m

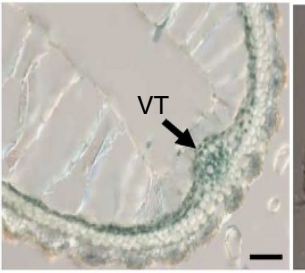

SCAMP1-REP

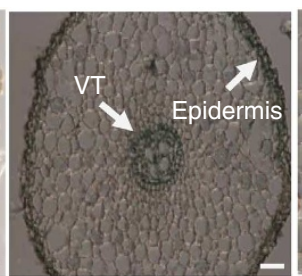

Merged

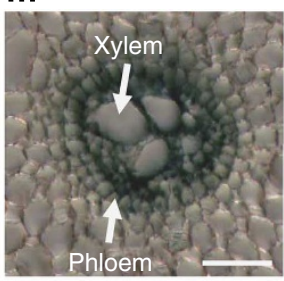

Bright

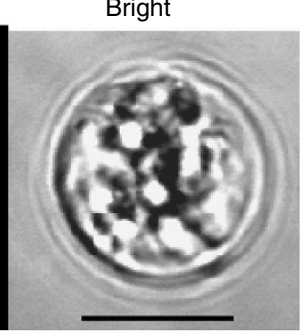

Bright
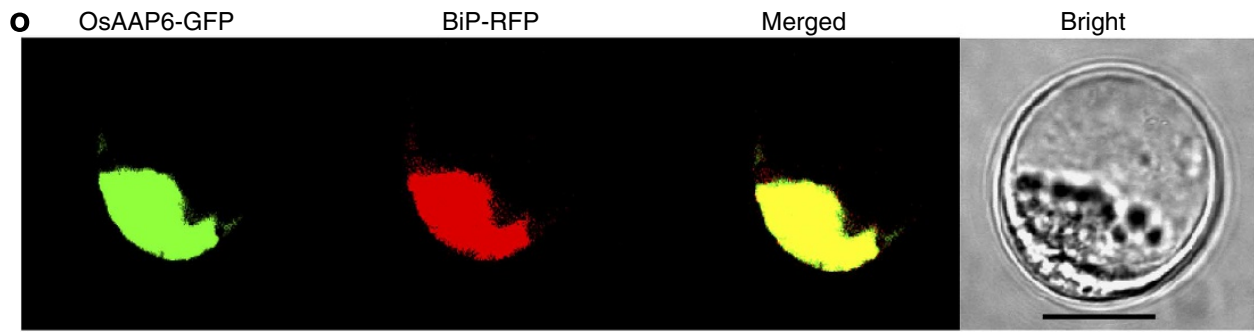

Figure 2 | Expression patterns of OsAAP6 and subcellular localization analysis. (a) The comparative expression pattern of OsAAP6 was determined by quantitative reverse transcription-PCR. RO, ST and FL: root, stem and flag leaf at the heading stage; YL, young leaf; 2YP, 4YP, 6YP, 8YP and 10YP: young panicles $2,4,6,8$ and $10 \mathrm{~cm}$ in length; $\mathrm{H} 4 \mathrm{H}$ and $\mathrm{HOH}$ : hulls at 4 and 0 days before heading; $\mathrm{H} 2 \mathrm{~A}$, hull at $2 \mathrm{DAF} ; 5 \mathrm{E}, 7 \mathrm{E}, 10 \mathrm{E}$ and $12 \mathrm{E}$ : endosperms at 5, 7, 10 and 12 DAF. All data are based on three biological replications. Error bars, s.e.m. (b-m) Representative histochemical analysis of tissue expression of GUS transgene under control of the OsAAP6 promoter from ZS97. (b) Endosperm 10 DAF. (c) Seed 10 DAF. (d) Seed 25 DAF. (e) Root. (f) Node. (g) Internode. (h) Hull at 2 days before flowering. (i) Pulvinus. (j,k) Hull cross sections. (I,m) Root cross sections. VT, vascular tissue; OVE, ovular vascular trace ends; OV, ovular vascular region; SV, lateral stylar vascular traces. Scale bars, $1 \mathrm{~mm}(\mathbf{j}), 50 \mu \mathrm{m}(\mathbf{k}-\mathbf{m})$. All transgenic plants were from the $\mathrm{T}_{1}$ generation, and at least ten independent transformants were subjected to histochemical GUS assays. (n,o) Subcellular localization analysis of OsAAP6. (n) OsAAP6-GFP does not co-localize with SCAMP1-RFP, which is located in the trans-Golgi network and plasma membrane, as a control. (o) Co-localization of OSAAP6-GFP and BiP-RFP, which is located in the ER. Merged images of YFP and bright-field images are also shown. Scale bar, $10 \mu \mathrm{m}$. 
a

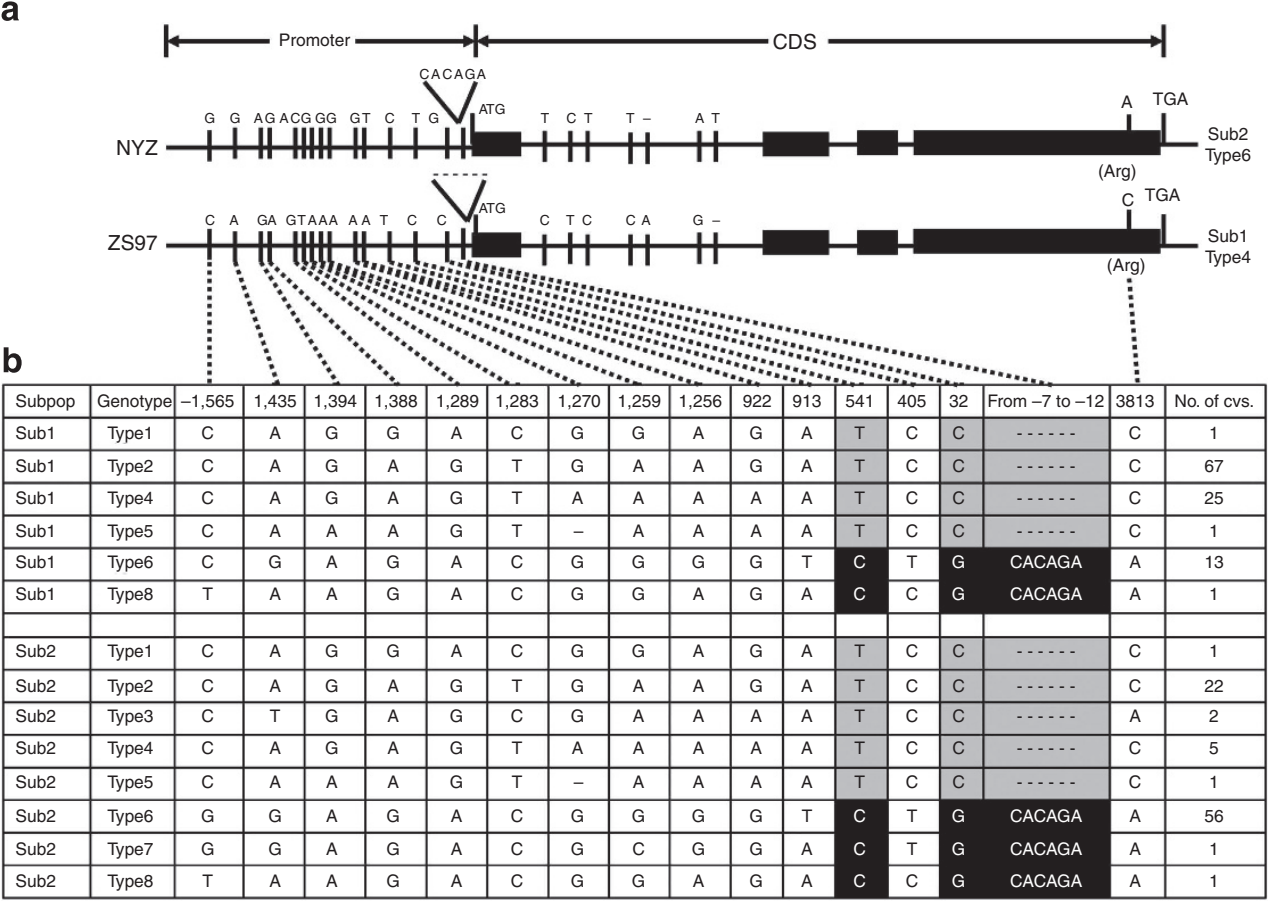

C

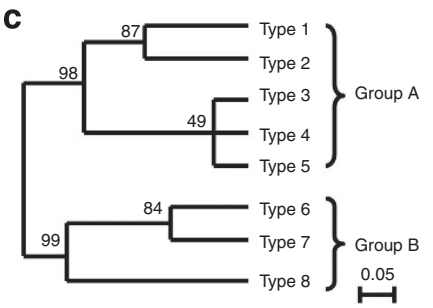

d

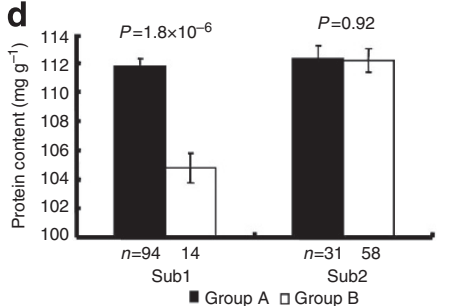

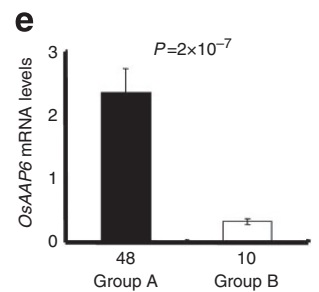

Group A Group B
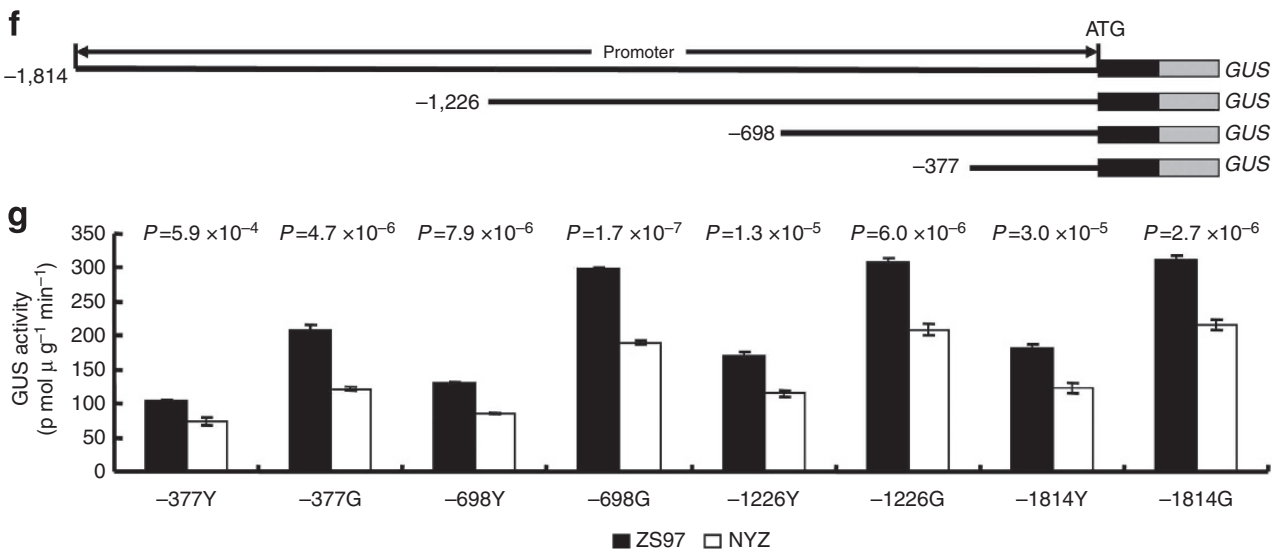

Figure 3 | Natural variation of OsAAP6 in a 197 accession rice mini-core collection and GUS activities in transgenic plants driven by eight promoter fragments and 5'-UTRs. (a) OsAAP6 gene structure and natural variation between alleles from ZS97 and NYZ. (b) Natural variation of OsAAP6 in 197 rice accessions of a mini-core collection compared with the NILs. (c) Cladogram of eight haplotypes. (d) Protein contents of brown rice in sub-populations $A$ and $B$; raw data are provided in Supplementary Table $5 ; n$, is the number of accessions. P-values were generated by two-tailed $t$-tests. Error bars, s.e.m. (e) OsAAP6 transcript levels in the endosperms of Sub1 cultivars with class A and B at 5 DAF; the number of accessions analysed is shown below each bar. The P-value was generated by a two-tailed $t$-test. Error bars, s.e.m. (f) Diagrams for the four deletions of the OsAAP6 promoter and $5^{\prime}$-UTR fused to the GUS gene. (g) Quantitative analysis of GUS activity in transgenic plants. Y and G indicate young panicles at 2 days before flowering and grains at $5 \mathrm{DAF}$, respectively. Data were from the transgenic lines planted in a randomized complete block design with three replications. $P$-values were produced by the Duncan test. Error bars denote s.e.m.

(Supplementary Fig. 5). These results strongly suggest that OsAAP6 enhances GPC by increasing grain storage protein (glutelins, prolamins, globulins and albumins) content and the total amount of amino acids, thus improving nutritional quality.
Changes in amino acids of root uptake and distribution. As OsAAP6 was preferentially expressed in the vascular tissues and rhizodermis of roots, we examined the effects of OsAAP6 on root uptake and distribution of various free amino acids. First, roots were submerged in a solution containing a mixture of 20 amino 
acids, and the depletion of each amino acid in this solution was measured $6 \mathrm{~h}$ after incubation. The study was not designed to establish actual rates of amino acid absorption by the plants, but rather to compare the NILs and transgenic plants with respect to this process. NIL(ZS97) and transgene-positive plants of $\mathrm{OX}(\mathrm{NYZ})$ had significantly increased threonine, serine, glycine, alanine, proline, acidic amino acids and total amino acids content, coupled with significantly reduced methionine, whereas the opposite was true for RNAi(ZS97) (Fig. 4a-c). The uptake data suggest that OsAAP6 greatly enhanced root absorption of a range of amino acids and displayed substantially higher uptake rates of threonine, serine, glycine, alanine, proline and acidic amino acids.

Uptake (or synthesis) of amino acids mainly takes place in mature roots and leaves; they are then exported via the stems to supply the flowers and grains. Sap flow in stems was analysed to determine whether OsAAP6 might play a role in this process. Serine, glycine, alanine, methionine, leucine, glutamate (or glutamine) and total amino acids levels were increased in NIL(ZS97) and transgene-positive plants of OX(NYZ), whereas lower levels were observed for RNAi(ZS97) (Fig. 4d-f).

Free amino acids in flag leaves and hulls were investigated to further determine whether OsAAP6 had any effect on the distribution of free amino acids. NIL(ZS97) and transgenepositive plants of OX(NYZ) showed substantial decreases in serine, alanine, valine, methionine, tyrosine, acidic amino acids and total content of free amino acids in flag leaves (at $10 \mathrm{DAF}$ ), whereas the opposite was true for the RNAi(ZS97) (Supplementary Fig. 6a-c). However, in hulls many free amino acids and total content of free amino acids were significantly increased in NIL(ZS97) and transgene-positive plants of $\mathrm{OX}(\mathrm{NYZ})$ (at $10 \mathrm{DAF}$ ), whereas the reverse was true for the RNAi(ZS97) (Supplementary Fig. 6d-f). Our results therefore suggest that OSAAP6 has effects on the distribution of various amino acids, at least in stems, flag leaves and hulls.

Pleiotropic effects of $\mathrm{OsAAP6}$ on gene expression. To determine whether changes in the accumulation of grain storage proteins and starch were reflected by altered messenger RNA levels, we examined the expression of key genes involved in grain storage materials. The mRNA transcript levels of 41 genes, including 20 involved in grain protein biosynthesis (Fig. 5a, I), 17 related to starch metabolism (12 involved in amylose biosynthesis (Fig. 5a, II) and 5 related to starch degradation (Fig. 5a, III)) in endosperms at 10 DAF were considerably upregulated in OX(NYZ) and NIL(ZS97), in contrast to plants not carrying the transgene and NIL(NYZ). The reverse was true for 37 of the 41 genes in RNAi(ZS97) (Fig. 5a). Furthermore, the transcript levels of three genes involved in amylopectin biosynthesis were markedly downregulated in NIL(ZS97) and OX(NYZ) transgenepositive plants, relative to control plants, and the reverse occurred for these three genes in RNAi(ZS97) (Fig. 5a, IV). These results
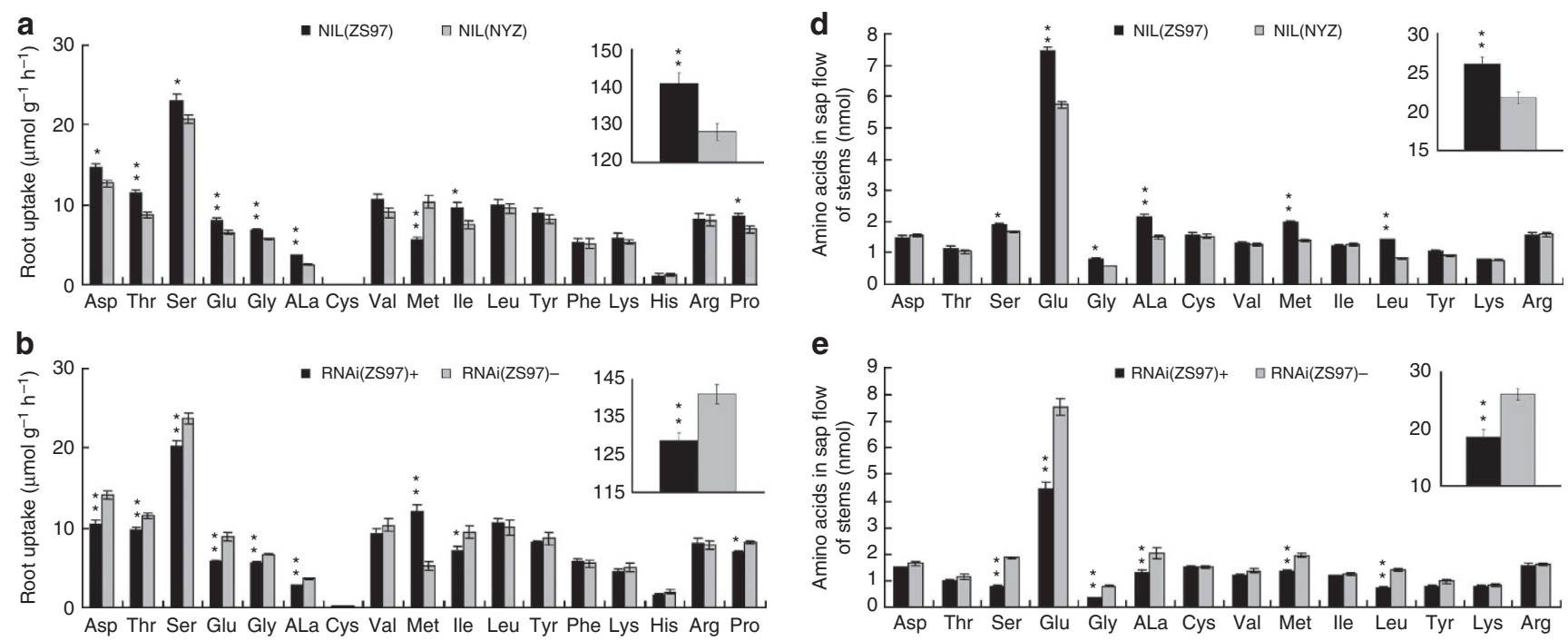

e
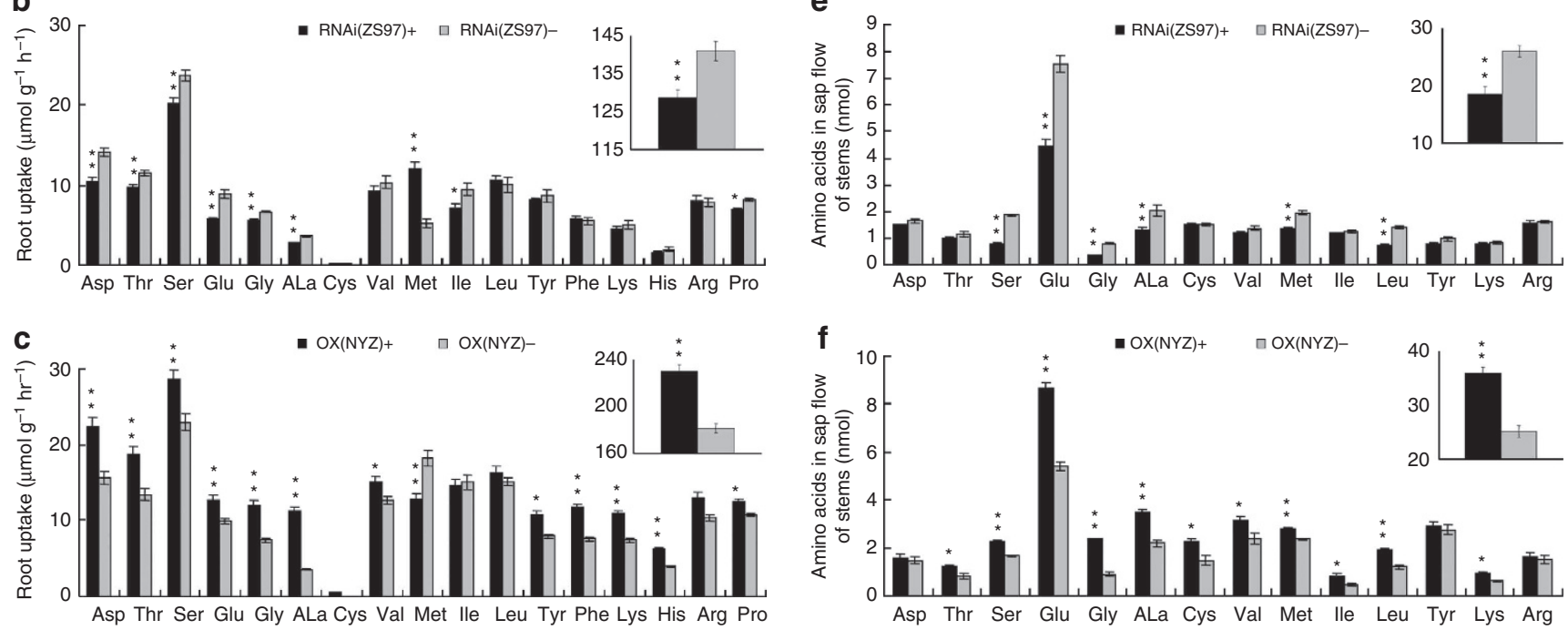

Figure 4 | Amino acid uptake and sap flow assays. (a-c) The effect of OSAAP6 on amino acid uptake by rice roots from NILs (a), RNAi (b) and OX(NYZ) (c). Plants were incubated in solutions containing a mixture of 20 amino acids, each at a concentration of $50 \mathrm{mM}$; rates of depletion of amino acids from this solution were determined. Root measurements were calculated using fresh weights. (d-f) Amino acid analysis of sap flow of stems from NILs (d), RNAi (e) and OX(NYZ) (f). Aliquots of $20 \mu$ l of stem sap were collected and assayed. Insert indicates the total content of amino acids. $(+)$ and $(-)$ indicate transgene-positive and negative $T_{2}$ plants, respectively. Significant differences at ${ }^{\star} P=0.05$ and ${ }^{\star \star} P=0.01$, respectively. All data are based on three biological replications and significant differences are based on two-tailed t-tests. Error bars, s.e.m. Glutamine (Gln) and asparagine (Asn) were hydrolysed to glutamate (Glu) and aspartate (Asp) under acidic conditions; thus, the final content of Glu was exactly the sum of the Gln and Glu contents and the final content of Asp was exactly the sum of the Asn and Asp contents. 

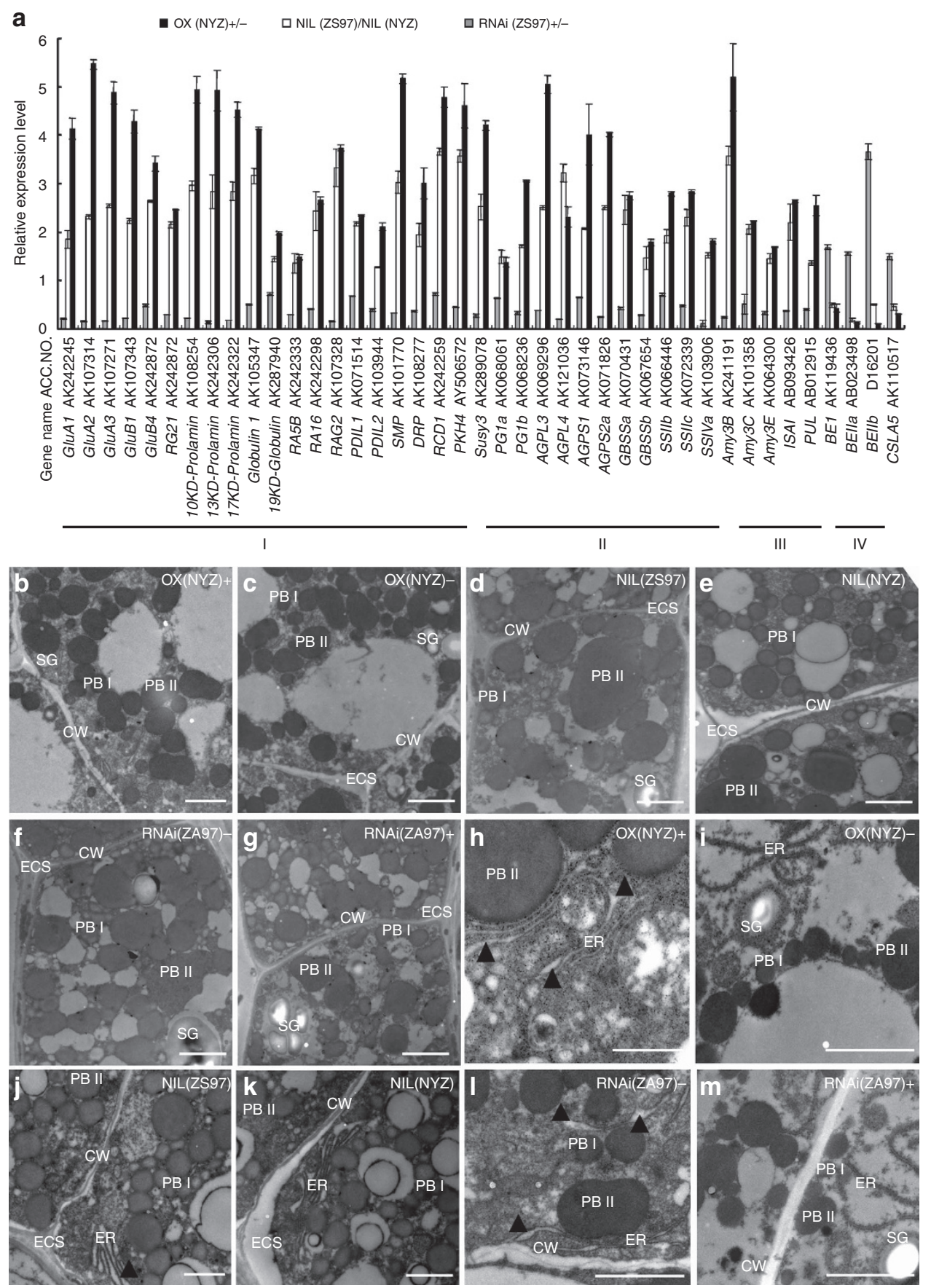

Figure 5 | Expression levels of genes involved in production of storage starch and proteins and transmission electron microscopy. (a) Expression levels of key genes involved on synthesis and storage of endosperm components in transgenic plants (OX(NYZ) and RNAi(ZS97)) in $T_{2}$ and NILs. Transgene-positive plants of OSAAP6 and NIL (ZS97) are shown relative to transgene-negative plants and NIL (NYZ), respectively, set as 1. Data are based on three biological replications. All $P$-values, produced by two-tailed $t$-tests, were $<0.01$. Error bar shows s.d. The annotated names of the genes are shown in Supplementary Table 7. (b-m) Ultrastructures of cells in developing endosperm of NILs and transgenic plants at 10 DAF. PBI and PBII, protein bodies I and II; SG, starch granule; ER, endoplasmic reticulum; ECS, extracellular space; CW, cell wall. Scale bars, $2 \mu \mathrm{m}$.

strongly suggest that OsAAP6 has significant effects on the expression of a large portion of the genes participating in starch and storage protein biosynthesis in developing rice grains.

To test whether changes at the transcriptional level affected enzyme behaviour, the activities of five key enzymes involved in starch metabolism were measured. At $10 \mathrm{DAF}$, NIL(ZS97) and transgene-positive plants of OX(NYZ) had increased activities of ADP-glucose pyrophosphorylase (ADPG-Pase), granule-bound starch synthase (GBSS), soluble starch synthase (SSS) and amylase, coupled with reduced starch branching enzyme (SBE) activity in developing endosperms, whereas the opposite was true for RNAi(ZS97) (Supplementary Fig. 7a-o). These results are consistent with the expression of key genes involved in starch metabolism (Fig. 5a) and the contents of amylose and starch (Table 1).

OsAAP6 enlarges protein bodies. As OsAAP6 greatly enhanced GPC in rice, transmission electron microscopy was employed to determine any effects on protein body (PB) formation. In 
developing endosperms (at $10 \mathrm{DAF}$ ), two types of PBs were readily discernible in ultra-thin sections; prolamin-containing PBI consistently shaped and surrounded by ER, and irregularly shaped glutelin/globulin-containing PBII showing uniform staining (Fig. 5b-m). In the developing endosperms of NIL(ZS97) and $\mathrm{OX}(\mathrm{NYZ})$, the mean section areas of PBI and PBII were all expanded, whereas the opposite was true for RNAi(ZS97) (Fig. 5b-g and Supplementary Fig. 8). Interestingly, the ER cisternal space was often dilated in transgene-positive plants of $\mathrm{OX}(\mathrm{NYZ})$ and NIL(ZS97), as well as transgene-negative plants of RNAi(ZS97) (Fig. 5h-m, black arrowheads). These results indicate that OsAAP6 has probable effects on the formation of $\mathrm{PBs}$ through enlargement.

\section{Discussion}

Rice grain quality is a complex character, and the demand for high-quality cereals as a source of protein has become increasingly evident ${ }^{4,41}$. Although numerous QTLs for GPC have been detected $^{17-22}$, no QTL controlling GPC underlying natural variation in rice has been cloned, and knowledge of the regulatory mechanism determining GPC was still lacking. In this study, we cloned a major QTL OsAAP6 that controls GPC by regulating the synthesis and accumulation of grain storage proteins and starch. Our results revealed that OsAAP6 functions as a positive regulator of GPC and greatly enhances the total amount of amino acids. Therefore, increasing the expression of OsAAP6 in low GPC rice varieties can increase GPC as well as the total amount of amino acids, and thus improve grain nutritional quality.

Amino acid transporters/permeases are key regulators of plant metabolism, growth and development ${ }^{24,25}$. The AAP family consists of eight members (AtAAP1-8) in Arabidopsis and 19 members $(O s A A P 1-19)$ in rice ${ }^{23}$. Our study showed that $q P C 1$ in rice corresponds to OsAAP6, which is highly homologous to AtAAP family genes (AtAAP1-8) in Arabidopsis and other putative genes in the staple cereals (Supplementary Fig. 9). Furthermore, comparison of the locations of QTLs controlling GPC with the 19 genes (OsAAP1-19) in rice indicated that $O s A A P 5$ and OsAAP8 are also located on the long arm of chromosome 1, whereas the other 16 OsAAPs are not closely associated with GPC QTLs ${ }^{22,23}$. Interestingly, OsAAP5 and OsAAP8 are barely expressed in seed ${ }^{23}$.

In plants, $A A P s$ appear to be involved in many physiological processes $^{23-25,42-45}$. The amount of protein was significantly decreased in desiccated seeds from AtAAP2 T-DNA insertion lines ${ }^{44}$. However, overexpression of VfAAP1 in both pea and Vicia narbonensis resulted in increased seed protein content ${ }^{46}$. Although the roles of many AtAAPs were revealed in Arabidopsis, no $O s A A P$ was functionally characterized in rice ${ }^{23}$. Here we isolated and characterized OsAAP6, and our results suggest that OsAAP6 has pleiotropic effects on grain storage materials (Table 1) and the expression of a large portion of the genes in developing rice grains (Fig. 5a).

In Arabidopsis, expression of AtAAPs is regulated by a complex array of cis-elements and transcription factors ${ }^{24,25}$. Sulphurresponsive element, copper-response element and inr element are targets for transcriptional activators/regulators and are involved in a broad range of responses ${ }^{34-40}$, including transcriptional activation/repression ${ }^{34-37}$, hormone signalling and fruit ripening ${ }^{38,39}$. Our results showed that the two common variations $(-7$ to $-12 \mathrm{bp},-32 \mathrm{bp}$ ) in the three potential cisregulatory elements seem to be associated with GPC diversity in indica cultivars (Fig. 3b-e and Supplementary Table 4), whereas there was no significant difference in GPC between Groups A and $\mathrm{B}$ in the japonica group using phenotypic data from years of 2010 and 2011 in Wuhan (Fig. 3d and Supplementary Table 5). This phenomenon may be due to the different genetic backgrounds between indica and japonica, and one gene is not possible to explain all the phenotype of GPC, which is controlled by many QTLs $^{17-22}$. Further investigation indicated that these potential cis-elements may play important roles in regulating the OsAAP6 expression difference between ZS97 and NYZ (Fig. 3f,g). Our results suggest that OsAAP6 expression may be regulated by the copper-responsive element, inr element and sulphur-responsive element separately or by joint interaction with transcription regulators. Although detailed studies of the cause of the GPC diversity need to be addressed, our results provide important information for identifying functional variations of OsAAP6.

Based on our results, we propose a model to explain the role of OsAAP6 in regulation of grain storage materials (Supplementary Fig. 10). We suggest that OsAAP6 functions as a positive regulator of GPC (Table 1, Supplementary Table 3 and Fig. 3). Increasing the expression level of OsAAP6 results in upregulation of key genes for storage proteins (Fig. 5a) and enhancement of root absorption of a range of amino acids (Fig. $4 \mathrm{a}-\mathrm{c}$ ), which may be distributed through the vascular tissues to the grains for biosynthesis and deposition of grain proteins (Figs $2 \mathrm{~b}-\mathrm{m}$ and $4 \mathrm{~d}-\mathrm{f}$, and Supplementary Fig. 6a-f), finally leading to larger PBs (Fig. 5b-g and Supplementary Fig. 8) and higher GPC (Table 1). OsAAP6 may indirectly effect the key genes for starch (amylose and amylopectin) biosynthesis (Fig. 5a, Supplementary Fig. 7a-o and Table 1).

Grain yield and quality are the two most important traits in rice research and breeding ${ }^{41}$, while GPC and amino acid composition are key factors determining the nutritional quality of cereal grains ${ }^{6}$. Breeding cereals with high protein while maintaining yield has been difficult ${ }^{2}$, because GPC is inherited as a typical polygenic trait ${ }^{1,17-21}$ that is sensitive to environmental factors ${ }^{17}$. Grain storage proteins are encoded by complex multigene families 5,10 and high GPC has negative effects on other traits ${ }^{2}$. Phylogenetic analysis of OsAAP6 revealed that it is highly homologous to the other putative genes in maize, sorghum, barley and wheat (Supplementary Fig. 9). Interestingly, OsAAP6 controlled GPC with no effect on plant morphology, flowering time or grain yield (Supplementary Table 6 and Supplementary Fig. 4), suggesting that GPC and nutritional quality could be improved without reduction in grain yield. Thus, map-based cloning and characterization of OsAAP6 have paved the way for genetic improvement of GPC and grain nutritional quality in rice and, potentially, other staple cereals.

\section{Methods}

Primers. Primers used in this study are listed in Supplementary Tables 7 and 8.

Map-based cloning of the qPC1 QTL. Two $\mathrm{BC}_{3} \mathrm{~F}_{2}$ populations consisting of 6,000 (population 1) and 4,008 (population 2) individuals were planted in the winter of 2008 on Hainan Island and in the summer of 2009 in an experimental field at Huazhong Agricultural University, respectively. To fine-map the $q P C 1$ locus, we developed new molecular markers (including SSR and InDel markers) from the sequences of $q P C 1$-flanking regions of Nipponbare and used them for genotyping All of the recombinant plant genotypes at the $q P C 1$ locus were determined by progeny testing in Wuhan.

Natural variation of OsAAP6 in a rice mini-core collection. We sequenced the genomic region of OsAAP6 in the NILs and 197 accessions of a rice mini-core collection, which included 101 cultivars of $O$. sativa ssp. indica and 96 cultivars of O. sativa spp. japonica originating from a wide geographic range across Asia. The population structure of the mini-core collection of 197 accessions was investigated by the model-based method implemented in STRUCTURE ${ }^{32,33}$. Total protein content of the grain was measured with an XDS Near-Infrared Rapid Content Analyzer (NIR, FOSS) by means of near-infrared reflectance spectroscopy ${ }^{47}$. Briefly, a Foss XDS spectrometer was used to obtain near-infrared spectral data 
between 400 and 2,500 $\mathrm{nm}$ at 2-nm intervals. The relationship between NIR spectral patterns of each sample and its GPC was examined, and data analysis was conducted using the WinISI software. The best predicted equation for GPC was selected on the basis of minimizing the s.e. of cross-validation and increasing the coefficient of determination, and the accuracy of model was evaluated on the validation subset, using coefficient of determination of the cross validation, slope and residual predictive value.

Plant growth conditions and trait measurement. Rice plants were examined under field conditions at the experimental stations of Huazhong Agricultural University in Wuhan and Hainan. The planting density was $16.5 \mathrm{~cm}$ between plants within rows and $26 \mathrm{~cm}$ between rows. Fields were managed according to local agricultural practices. Harvested rice grains were air-dried and stored at room temperature for at least 3 months before testing. Fully filled grains were used for measuring grain quality and yield traits. Milled rice was ground to flour for measurement of amylose content, starch content and gel consistency ${ }^{48}$. Glutelins, prolamins, globulins and albumins were extracted sequentially from seeds using three solvents, and quantitative analyses of the proteins were executed by TECAN Infinite M200 (refs 15,49). Grain weights determined on samples of 200 grains were converted to 1,000-grain weights.

RNA preparation and reverse transcription. Total RNA was extracted from various plant tissues with an RNA extraction kit and TRIzol reagent (Invitrogen). First-strand cDNA was synthesized with $2 \mu \mathrm{g}$ of RNA and $200 \mathrm{U}$ of M-MLV reverse transcriptase (Promega) in a volume of $20 \mu \mathrm{l}$.

Constructs and transformation. To prepare the complementation construct $(\mathrm{ZpZc})$, we fused the 1.8 -kb promoter fragment and $5^{\prime}$-UTR of OsAAP6 from ZS97 with the coding region from ZS97; the OsAAP6 promoter, $5^{\prime}$-UTR and coding region were obtained by PCR from ZS97 and confirmed by sequencing. The coding region from ZS97 was first inserted into the plant binary vector pCAMBIA1301, and then the promoter fragment and $5^{\prime}$-UTR was fused with it. To prepare the overexpression (OX) construct, we inserted the OsAAP6 coding region of ZS97 into the plant binary vector pCAMBIA1301S under control of the CaMV 35S promoter. To generate the RNAi construct, we cloned a PCR fragment containing $580 \mathrm{bp}$ from the fourth exon of OsAAP6 (nucleotides 3,364-3,943) into the dspCAMBIA1301 vector in both the sense and the antisense orientations. The OX construct was introduced into the rice cultivars NYZ (OX(NYZ)) and ZH11 (OX(ZH11)), and $\mathrm{ZpZc}$ was introduced into NYZ $(\mathrm{ZpZc}(\mathrm{NYZ})$ ) by means of Agrobacteriummediated transformation. We introduced the RNAi construct by means of Agrobacterium-mediated transformation into ZS97 and ZH11 (RNAi(ZS97) and RNAi(ZH11), respectively) by means of a previously described procedure ${ }^{50,51}$, with minor modifications (each subculture was conducted on a subculture medium at $25^{\circ} \mathrm{C}$ in the dark for 18 days). The $5^{\prime}$ deletions of the OsAAP6 promoter from ZS97 and NYZ at positions $-1,814,-1,226,-698$ and -377 were generated by PCR amplification and inserted into expression vector pDX2181 (ref. 52). The resulting constructs (designated as $-1,814,-1,226,-698$ and -377 from ZS97 and NYZ4, respectively) were introduced into Agrobacterium tumefaciens strain EHA105 and transferred into ZH11 by Agrobacterium-mediated transformation ${ }^{50}$.

Quantitative real-time PCR. Quantitative real-time PCR was carried out in a total volume of $25 \mu \mathrm{l}$ containing $2 \mu \mathrm{l}$ of the reverse-transcribed product, $0.2 \mathrm{mM}$ of genespecific primers, $12.5 \mu \mathrm{l}$ of SYBR Premix EX Taq and $0.5 \mu \mathrm{l}$ of Rox Reference Dye II (Takara) on an ABI 7500 real-time PCR system, according to the manufacturer's instructions. Measurements were obtained by means of the relative quantification method $^{53}$. The rice Actinl gene was used as the internal control. All expression level data obtained by quantitative real-time PCR are based on at least two biological samples on which three replications of the technique were conducted.

Histochemical and fluorometric GUS assays. GUS activity in the various tissues of transgenic-positive plants was localized histochemically ${ }^{52}$, and photographs were taken under a dissecting microscope. Tissues of transgenic plants were incubated overnight in GUS staining solution at $37^{\circ} \mathrm{C}$, dehydrated and embedded in paraffin (Sigma). The tissues were sliced into $10-\mu \mathrm{m}$ sections with a microtome and fixed to microscope slides and examined under a fluorescence microscope. For each construct, at least ten independent transformants were subjected to histochemical GUS assays. The protein concentration in the supernatant was quantified by means of the Bradford assay ${ }^{49}$. GUS protein concentration in the supernatant was determined fluorometrically with an INFINITE 200 photometer (Tecan Austria Gmbh). For each construct, 15 GUS staining-positive transformants were used for quantitative analysis of GUS activity. Three biological replicates per construct were analysed by fluorometric assays and averages were calculated.

Assays of enzyme activities. Endosperms of 50 dehulled grains were separated from the embryos and pericarps, and homogenized in a pre-cooled mortar containing $5 \mathrm{ml}$ frozen extraction medium. The homogenate was centrifuged at $12,000 \mathrm{~g}$ for $20 \mathrm{~min}$ at $4{ }^{\circ} \mathrm{C}$, the extracted solution was used for measuring enzyme activities and the precipitate was used for measuring the granule-bound starch synthase activity, which was assayed by TECAN Infinite M200 (refs 54,55). The extracted solution was assayed for the activities of ADPG-Pase, SSS, amylase and $\mathrm{SBE}^{56}$. The activities of ADPG-Pase and SSS were defined as the NADPH amount by measuring increases in absorbance at $340 \mathrm{~nm}$, and SBE activity was defined as the decrease in amount of $1 \% \mathrm{KI}_{-} \mathrm{I}_{2}$ at $540 \mathrm{~nm}$. Three replications were conducted for every experiment.

Subcellular localization of the OsAAP6 protein. The full-length OsAAP6 cDNA was amplified by PCR using cDNA obtained from reverse transcription. The amplified OsAAP6 cDNA fragment was inserted into a premade GFP vector in pM999 via SacI and XbaI restriction enzyme sites to generate OsAAP6-GFP, and PEG-mediated transfections were carried out ${ }^{57}$. Well-established fluorescent protein markers BiP-RFP for the $\mathrm{ER}^{28}$ and SCAMP1-RFP for the trans-Golgi network and plasma membrane $e^{29-31}$ were used. Protoplasts were observed using a confocal laser scanning microscope and visualized by a Leica Microsystem LAS AF. All fluorescence experiments were independently repeated at least three times.

Transmission electron microscopy analyses. Transverse sections (1-mm thick) of developing seeds were fixed for over $12 \mathrm{~h}$ in $2.5 \%$ glutaraldehyde buffered with $0.2 \mathrm{M}$ phosphate buffer ( $\mathrm{pH}$ 7.2). The sections were treated and sectioned with an ultramicrotome (Power Tome-XL, RMC). Microscopic observation was performed using a transmission electron microscope (H-7650, Hitachi). The area of PBs in each sample was determined using ImageJ software (NIH).

Amino acid analysis. NILs and transgenic plants were grown in a rice culture medium ${ }^{50}$ under sterile conditions and placed in a climate chamber with a 14 -h light/10-h darkness and $25^{\circ} \mathrm{C} / 18^{\circ} \mathrm{C}$ (day/night) regime. After 20 days of growth, 3 plants of each plant type were selected for the uptake experiment, which was carried out on a shaking table in the climate chamber. The uptake solution was identical to the rice culture solution used during the plant cultivation, except that all $\mathrm{N}$ sources were replaced with a 20 -amino-acid mixture composed of equal amounts of $50 \mathrm{mM}$ solutions for each. Root uptake rates were determined from the decline in concentration of each amino acid in the solution during the $6 \mathrm{~h}$ incubation. The amino acid concentrations were determined using an automatic amino acid analyser of L-8800 (ref. 58).

Amino acid contents in the stem sap flow were obtained 10 DAF. Plants were cut $10 \mathrm{~cm}$ above the roots. Aliquots of $20 \mu \mathrm{l}$ sap from the remaining stem bases were collected for $30 \mathrm{~min}$ after discarding the first drops, then lyophilized and re-suspended in $480 \mu \mathrm{l} 0.02 \mathrm{M} \mathrm{HCL}$ and analysed by an L- 8800 automatic amino acid analyser 58

Free amino acids in flag leaves and hulls, also at $10 \mathrm{DAF}$, were separately extracted with $40 \mathrm{ml}$ of $80 \%$ ethanol. Aliquots $(1 \mathrm{ml})$ of each sample were evaporated and the residues were re-dissolved in $1 \mathrm{ml}$ of $0.02 \mathrm{M} \mathrm{HCl}$ and analysed with an L-8800 automatic amino acid analyser ${ }^{58}$.

\section{References}

1. Uauy, C., Distelfeld, A., Fahima, T., Blech, A. \& Dubcovsky, J. A NAC gene regulating senescence improves grain protein, zinc, and iron content in wheat Science 314, 1298-1301 (2006).

2. Shewry, P. R. Improving the protein content and composition of cereal grains J. Cereal Sci. 46, 239-250 (2007).

3. Balyan, H. S. et al. Genetic improvement of grain protein content and other health-related constituents of wheat grain. Plant Breed. 132, 446-457 (2013).

4. Fitzgerald, M. A., McCouch, S. R. \& Hall, R. D. Not just a grain of rice: the quest for quality. Trends Plant Sci. 14, 133-139 (2009).

5. Tian, Z. X. et al. Allelic diversities in rice starch biosynthesis lead to a diverse array of rice eating and cooking qualities. Proc. Natl Acad. Sci. USA 106, 21760-21765 (2009).

6. Ufaz, S. \& Galili, G. Improving the content of essential amino acids in crop plants: goals and opportunities. Plant Physiol. 147, 954-961 (2008).

7. Juliano, B. O. Structure, chemistry, and function of the rice grain and its fractions. Cereal Foods World 37, 772-774 (1992).

8. Shewry, P. R., Napier, J. A. \& Tatham, A. S. Seed storage protein: structure and biosynthesis. Plant Cell 7, 945-956 (1995).

9. Zhang, W. et al. QTL mapping for crude protein and protein fraction contents in rice (Oryza sativa L.). J. Cereal Sci. 48, 539-547 (2008).

10. Duan, M. J. \& Sun, S. S. M. Profiling the expression of genes controlling rice grain quality. Plant Mol. Biol. 59, 165-178 (2005).

11. Kawakatsu, T., Hirose, S., Yasuda, H. \& Takaiwa, F. Reducing rice seed storage protein accumulation leads to changes in nutrient quality and storage organelle formation. Plant Physiol. 154, 1842-1854 (2010).

12. Wang, G. et al. Opaque7 encodes an acyl-activating enzyme-like protein that affects storage protein synthesis in maize endosperm. Genetics 189, 1281-1295 (2011). 
13. She, K. et al. A novel factor FLOURY ENDOSPERM2 is involved in regulation of rice grain size and starch quality. Plant Cell 22, 3280-3294 (2010).

14. Wang, Y. H. et al. OsRab5a regulates endomembrane organization and storage protein trafficking in rice endosperm cells. Plant J. 64, 812-824 (2010).

15. Iida, S., Amano, E. \& Nishio, T. A rice (Oryza sativa L.) mutation having a low content of glutelin and a high content of prolamine. Theor. Appl. Genet. 87, 374-378 (1993).

16. Holding, D. R. et al. The maize floury1 gene encodes a novel endoplasmic reticulum protein involved in zein protein body formation. Plant Cell 19, 2569-2582 (2007).

17. Tan, Y. F. et al. Mapping quantitative trait loci for milling quality, protein content and color characteristics of rice using a recombinant inbred line population derived from an elite rice hybrid. Theor. Appl. Genet. 103, 1037-1045 (2001).

18. Aluko, G. et al. QTL mapping of grain quality traits from the interspecific cross Oryza sativa $\times$ O. glaberrima. Theor. Appl. Genet. 109, 630-639 (2004).

19. Lou, J. et al. QTL mapping of grain quality traits in rice. J. Cereal Sci. 50, 145-151 (2009).

20. Ye, G. Y., Liang, S. S. \& Wan, J. M. QTL mapping of protein content in rice using single chromosome segment substitution lines. Theor. Appl. Genet. 121, 741-750 (2010).

21. Yu, Y. H. et al. Genetic relationship between grain yield and the contents of protein and fat in a recombinant inbred population of rice. J. Cereal Sci. 50, 121-125 (2009)

22. Wang, L. Q. et al. The QTL controlling amino acid content in grains of rice (Oryza sativa) are co-localized with the regions involved in the amino acid metabolism pathway. Mol. Breed. 21, 127-137 (2008).

23. Zhao, H. M., Ma, H. L., Yu, L., Wang, X. \& Zhao, J. Genome-wide survey and expression analysis of amino acid transporter gene family in rice (Oryza sativa L.). PLoS ONE 7, e49210 (2012).

24. Tegeder, M. Transporters for amino acids in plant cells: some functions and many unknowns. Curr. Opin. Plant Biol. 15, 315-321 (2012).

25. Liu, X. \& Bush, D. R. Expression and transcriptional regulation of amino acid transporters in plants. Amino Acids 30, 113-120 (2006).

26. Svennerstam, H., Ganeteg, U., Bellini, C. \& Nasholm, T. Comprehensive screening of Arabidopsis mutants suggests the Lysine Histidine Transporter 1 to be involved in plant uptake of amino acids. Plant Physiol. 143, 1853-1860 (2007).

27. Hirner, A. et al. Arabidopsis LHT1 is a high-affinity transporter for cellular amino acid uptake in both root epidermis and leaf mesophyll. Plant Cell $\mathbf{1 8}$ 1931-1946 (2006).

28. Min, M. K. et al. Overexpression of Arabidopsis AGD7 causes relocation of Golgi-localized proteins to the endoplasmic reticulum and Inhibits protein trafficking in plant cells. Plant Physiol. 143, 1601-1614 (2007).

29. Lam, S. K. et al. Rice SCAMP1 defines clathrin-coated, trans-Golgi-located tubular-vesicular structures as an early endosome in tobacco BY-2 cells. Plant Cell 19, 296-319 (2007).

30. Lam, S. K., Cai, Y., Hillmer, S., Robinson, D. G. \& Jiang, L. W. SCAMPs highlight the developing cell plate during cytokinesis in tobacco BY-2 cells. Plant Physiol. 147, 1637-1645 (2008).

31. Wang, J. et al. EXPO, an exocyst-positive organelle distinct from multivesicular endosomes and autophagosomes, mediates cytosol to cell wall exocytosis in Arabidopsis and tobacco cells. Plant Cell 22, 4009-4030 (2010).

32. Pritchard, J. K., Stephens, M. \& Donnelly, P. Inference of population structure using multilocus genotype data. Genetics 155, 945-959 (2000).

33. Agrama, H. A., Eizenga, G. C. \& Yan, W. Association mapping of yield and its components in rice cultivars. Mol. Breed. 19, 341-356 (2007).

34. Okushima, Y. et al. Functional genomic analysis of the AUXIN RESPONSE FACTOR gene family members in Arabidopsis thaliana: unique and overlapping functions of ARF7 and ARF19. Plant Cell 17, 444-463 (2005).

35. Ulmasov, T., Hagen, G. \& Guilfoyle, T. J. Activation and repression of transcription by auxin- response factors. Proc. Natl Acad. Sci. USA 96, 5844-5849 (1999).

36. Kropat, J. et al. A regulator of nutritional copper signaling in Chlamydomonas is an SBP domain protein that recognizes the GTAC core of copper response element. Proc. Natl Acad. Sci. USA 102, 18730-18735 (2005).

37. Quinn, J. M. \& Merchant, S. Two copper-responsive elements associated with the Chlamydomonas Cyc6 gene function as targets for transcriptional activators. Plant Cell 7, 623-628 (1995).

38. Hou, H. M. et al. Genomic organization, phylogenetic comparison and differential expression of the SBP-Box family genes in grape. PLOS ONE 8, e59358 (2013)

39. Manning, K. et al. A naturally occurring epigenetic mutation in a gene encoding an SBP-box transcription factor inhibits tomato fruit ripening. Nat. Genet. 38, 948-952 (2006).

40. Nakamura, M., Tsunoda, T. \& Obokata, J. Photosynthesis nuclear genes generally lack TATA-boxes: a tobacco photosystem I gene responds to light through an initiater. Plant J. 29, 1-10 (2002).
41. Zhang, Q. F. Strategies for developing green super rice. Proc. Natl Acad. Sci. USA 104, 16402-16409 (2007).

42. Svennerstam, H., Ganeteg, U. \& Näsholm, T. Root uptake of cationic amino acids by Arabidopsis depends on functional expression of amino acid permease 5. New Phytol. 180, 620-630 (2008).

43. Sanders, A. et al. AAP1 regulates import of amino acids into developing Arabidopsis embryos. Plant J. 59, 540-552 (2009).

44. Zhang, L. L. et al. Altered xylem-phloem transfer of amino acids affects metabolism and leads to increased seed yield and oil content in Arabidopsis. Plant Cell 22, 3603-3620 (2010).

45. Schmidt, R., Stransky, H. \& Koch, W. The amino acid permease AAP8 is important for early seed development in Arabidopsis thaliana. Planta 226, 805-813 (2007).

46. Rolletschek, H. et al. Ectopic expression of an amino acid transporter (VfAAP1) in seeds of Vicia narbonensis and pea increases storage proteins. Plant Physiol. 137, 1236-1249 (2005).

47. Perbandt, D., Reulein, J., Richter, F., Reinhold, S. \& Wachendorf, M. Assessment of mass flows and fuel quality during mechanical dehydration of silages using near infrared reflectance spectroscopy. Bioenerg. Res. 3, 194-203 (2010).

48. Tan, Y. F. et al. The three important traits for cooking and eating quality of rice grains are controlled by a single locus in an elite rice hybrid, Shanyou 63. Theor. Appl. Genet. 99, 642-648 (1999).

49. Bradford, M. A rapid and sensitive method for the quantitation of microgram quantities of protein utilizing the principle of protein-dye binding. Anal. Biochem. 72, 248-254 (1976).

50. Hiei, Y., Ohta, S., Komari, T. \& Kumashiro, T. Efficient transformation of rice (Oryza sativa L.) mediated by Agrobacterium and sequence analysis of the boundaries of the T-DNA. Plant J. 6, 271-282 (1994).

51. Lin, Y. J. \& Zhang, Q. F. Optimizing the tissue culture conditions for high efficiency transformation of indica rice. Plant Cell Rep. 23, 540-547 (2005).

52. Ye, R. J., Zhou, F. \& Lin, Y. J. Two novel positive cis-regulatory elements involved in green tissue-specific promoter activity in rice (Oryza sativa $\mathrm{L}$ ssp.). Plant Cell Rep. 31, 1159-1172 (2012).

53. Livak, K. \& Schmittgen, T. Analysis of relative gene expression data using realtime quantitative PCR and the $2^{-\Delta \Delta C T}$ method. Methods 25, 402-408 (2001).

54. Umemoto, T., Nakamura, Y. \& Ishikura, N. Activity of starch synthase and the amylose content in rice endosperm. Phytochem. 40, 1613-1616 (1995).

55. Umemoto, T. \& Terashima, K. Activity of granule-bound starch synthase is an important determinant of amylose content in rice endosperm. Funct. Plant Biol. 29, 1121-1124 (2002).

56. Nakamura, Y., Yuki, K., Park, S. Y. \& Ohya, T. Carbohydrate metabolism in the developing endosperm of rice grains. Plant Cell Physiol. 30, 833-839 (1989).

57. Zhang, Y. et al. A highly efficient rice green tissue protoplast system for transient gene expression and studying light/chloroplast-related processes. Plant Methods 7, 30 (2011).

58. Zhou, Y. et al. Over-expression of aspartate aminotransferase genes in rice resulted in altered nitrogen metabolism and increased amino acid content in seeds. Theor. Appl. Genet. 118, 1381-1390 (2009).

\section{Acknowledgements}

We thank Dr Qifa Zhang (Huazhong Agricultural University) and Dr John Bennett (School of Biological Sciences, University of Sydney) for constructive suggestions and critical reading of the manuscript. This work was supported by grants from the National 863 Project (2012AA10A303), the National Program on R\&D of Transgenic Plants (2014ZX08009-036B, 2013ZX08009003-004), the National Natural Science Foundation (31171523), the National Program on the Development of Basic Research (2011CB100200) and the National Agricultural Industry Technique System of China (CARS-01-03).

\section{Author contributions}

B.P. conducted most of the experiments, including fine mapping, gene cloning, expression analysis, genetic transformation, quality traits analysis, rice germplasm analysis, histochemical assays, quantitative analyses of GUS activity, promoter analysis, enzyme activities analyses and other functional analyses. L.J.L. constructed the segregating backcross populations; M.Z. and L.W. conducted the QTL primary mapping analysis. H.K. developed the NILs and participated in part of fine mapping. Y.L. carried out part of the expression analysis; G.W. and X.L. provided rice germplasm samples; W.X. and W.Y. carried out part of nucleotide diversity analysis; J.C. participated in part of the quality traits analyses. L.S., G.G., Q.Z., J.X., C.X. and X.L. participated in the promoter sequencing and rice germplasm analysis. Y.P. and L.L. participated in part of the enzyme activities analyses. Y.H. designed and supervised the study. Y.H. and B.P. analysed the data and wrote the paper.

\section{Additional information}

Accession codes: Genomic DNA of OsAAP6 in Zhenshan 97 has been deposited in GenBank/EMBL/DDBJ under the accession code KM213629. Genomic DNA of OsAAP6 
in Nanyangzhan has been deposited in GenBank/EMBL/DDBJ under the accession code KM213630.

Supplementary Information accompanies this paper at http://www.nature.com/ naturecommunications

Competing financial interests: The authors declare no competing financial interests.

Reprints and permission information is available online at http://npg.nature.com/ reprintsandpermissions/
How to cite this article: Peng, B. et al. OsAAP6 functions as an important regulator of grain protein content and nutritional quality in rice. Nat. Commun. 5:4847 doi: 10.1038/ncomms5847 (2014).

(c) (i) This work is licensed under a Creative Commons Attribution 4.0 International License. The images or other third party material in this article are included in the article's Creative Commons license, unless indicated otherwise in the credit line; if the material is not included under the Creative Commons license, users will need to obtain permission from the license holder to reproduce the material. To view a copy of this license, visit http://creativecommons.org/licenses/by/4.0/ 